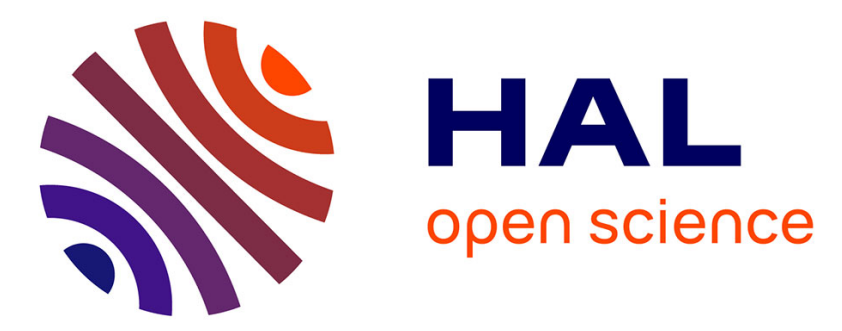

\title{
Three-dimensional structure of the mammalian limbal stem cell niche
}

Kate Grieve, Djida Ghoubay, Cristina Nico Georgeon, Olivier Thouvenin, Nacim Bouheraoua, Michel Paques, Vincent M Borderie

\section{- To cite this version:}

Kate Grieve, Djida Ghoubay, Cristina Nico Georgeon, Olivier Thouvenin, Nacim Bouheraoua, et al.. Three-dimensional structure of the mammalian limbal stem cell niche. Experimental Eye Research, 2015, 140, pp.75-84. 10.1016/j.exer.2015.08.003 . hal-01191771

\section{HAL Id: hal-01191771 \\ https://hal.sorbonne-universite.fr/hal-01191771}

Submitted on 2 Sep 2015

HAL is a multi-disciplinary open access archive for the deposit and dissemination of scientific research documents, whether they are published or not. The documents may come from teaching and research institutions in France or abroad, or from public or private research centers.
L'archive ouverte pluridisciplinaire HAL, est destinée au dépôt et à la diffusion de documents scientifiques de niveau recherche, publiés ou non, émanant des établissements d'enseignement et de recherche français ou étrangers, des laboratoires publics ou privés.

\section{(1) (1) $\$$}

Distributed under a Creative Commons Attribution - NonCommercial - NoDerivatives| 4.0 


\title{
Three-dimensional structure of the mammalian limbal stem cell niche
}

\author{
K. Grieve a, b, *, D. Ghoubay a, b , C. Georgeon ${ }^{\text {a }}$, O. Thouvenin ${ }^{\text {c }}$, N. Bouheraoua ${ }^{\text {a, b }}$, \\ M. Paques a, b, V.M. Borderie a, b \\ a Quinze Vingts National Ophthalmology Hospital, 28 rue de Charonne, 75012 Paris, France \\ b Vision Institute, UPMC Université Paris 06, UMR_S 968/INSERM, U968/CHNO des XV-XX/CNRS, UMR_7210, France \\ ${ }^{\mathrm{c}}$ Institut Langevin, 1 rue Jussieu, 75005 Paris, France
}

\section{A R T I C L E I N F O}

\section{Article history:}

Received 5 March 2015

Received in revised form

24 July 2015

Accepted in revised form 4 August 2015

Available online 20 August 2015

\section{Keywords:}

Corneal limbus

Stem cells

Three-dimensional imaging

Optical coherence tomography

Cornea

\begin{abstract}
A B S T R A C T
Although the existence of the limbal stem cell (LSC) niche is accepted, precise knowledge of its threedimensional (3D) architecture remains incomplete. The LSC niche was explored on freshly excised and organ-cultured corneoscleral rims from human donors $(n=47)$, pigs $(n=15)$ and mice $(n=27)$ with full-field optical coherence microscopy (FFOCM). Limbal crypt features were detected in $90 \%$ of organ-cultured human corneoscleral rims, extending between the palisades of Vogt as radially oriented rectangular (74\% of eyes) and/or rounded (23\% of eyes) forms, often branching off to, or becoming interconnected by, sub-scleral radially or circumferentially oriented crypts (in $56 \%$ of eyes). Mean crypt volume represented $16 \%$ of sampled limbal volume on the vertical axis and $8 \%$ on the horizontal axis. In pigs, palisades were finer and crypts wider with relatively uniform distribution around the eye, and radial orientation, connecting to numerous narrow criss-crossing invaginations beneath the scleral surface. In mice, only a circumferential limbal trough was detected. Mean crypt volume represented $13 \%$ of sampled limbal volume in humans and $9 \%$ in pigs. FFOCM combined with fluorescence, and confocal fluorescence microscopy, showed presence of p63- $\alpha+$ cells and cytokeratin-3+ cells in the limbal crypts. To assess colony forming efficiency (CFE), limbal epithelial cells were cultured at low density with mitomycin-arrested 3T3 feeders. CFE increased with limbal crypt volume and was not significantly decreased in organ-cultured cornea, despite degradation of the epithelial roof, suggesting that stem cells remain protected at the base of crypts during organ culture. CFE in human samples was significantly greater than in pig, and CFE in mouse was zero. Crypt architecture in the three species appears associated with eye exposure to light. LSC density increased with percentage limbal volume occupied by crypts.
\end{abstract}

(c) 2015 The Authors. Published by Elsevier Ltd. This is an open access article under the CC BY-NC-ND license (http://creativecommons.org/licenses/by-nc-nd/4.0/).

\section{Introduction}

The limbal stem cell niche, situated at the anatomic border of the conjunctiva and the cornea (Levis et al., 2013; Eberwein and Reinhard, 2014, Rama et al., 2010), provides a microenvironment that contributes to the development and maintenance of the

Abbreviations: 3D, three-dimensional; 2D, two-dimensional; OCT, optical coherence tomography; LSC, limbal stem cell; FFOCM, full-field optical coherence microscopy; LEC, limbal epithelial crypts; LC, limbal crypts; CFE, colony forming efficiency; CFA, colony forming assay; PBS, phosphate buffered solution; PFA, paraformaldehyde; CMOS, complementary metal oxide semiconductor; LED, light emitting diode; UV, ultraviolet.

* Corresponding author. Institut de la Vision Centre Hospitalier National d'Ophtalmologie des Quinze-Vingts, 17 rue Moreau, 75012 Paris, France.

E-mail address: kategrieve@gmail.com (K. Grieve). extracellular matrix, resident cells, and their signals that define a stem cell niche (Eberwein and Reinhard, 2014, Dua et al., 2005). The distinctive protected environment of the corneoscleral limbus features dense vascularization, innervation, and protection from potential light damage thanks to the presence of melanin pigmentation (Davanger and Evensen, 1971; Goldberg and Bron, 1982 and Townsend, 1991). The corneal transition and limbal palisade regions appear to control growth factor signaling in order to provide a unique microenvironment for corneal epithelial stem and progenitor cells. While constituents of the stem cell compartment in the posterior limbus may provide extrinsic signals that contribute to maintenance of stemness (Tseng, 1996; Morrison et al., 1997; Watt and Hogan, 2000; Schlötzer-Schrehardt et al., 2005, 2007), components of the late progenitor cell compartment in the anterior limbus may regulate the phenotypic changes that 
are necessary to repopulate the regenerating corneal epithelium (Schlötzer-Schrehardt et al., 2007).

The existence of the limbal niche is accepted, but precise knowledge of its 3D architecture remains elusive (Dziasko et al., 2014). Exploration of the architecture of the corneal limbus and the limbal crypts has been carried out using different techniques, with different structures found (Goldberg and Bron, 1982; Townsend, 1991; Dua et al., 2005; Shortt et al., 2007; Shanmuganathan et al., 2007; Miri et al., 2012; Lathrop et al., 2012; Molvær et al., 2013). Both Goldberg and Bron (1982) and Townsend (1991) examined the corneal limbus with a slit lamp and described the palisades of Vogt as a series of radially oriented fibrovascular ridges, concentrated along the upper and lower corneoscleral limbus, separated by interpalisade epithelial rete ridges. They found high variability of the palisade zone from one individual to another and within the same eye, along with high variability of the shape of palisades and interpalisade epithelial crypts, including radially oriented rectangular and/or circular or oval forms, and branching or interconnection of the palisades to produce a trabecular pattern. Indeed, the high variability led Goldberg and Bron (1982) to conclude that the limbal palisade pattern is as individual as a fingerprint. Limbal crypt structure has been explored using histology (Dua et al., 2005; Shanmuganathan et al., 2007; Nubile et al., 2013; Molvær et al., 2013), confocal fluorescence microscopy (Shortt et al., 2007; Yeung et al., 2008), electron microscopy (Dua et al., 2005; Shanmuganathan et al., 2007; Shortt et al., 2007) in vivo confocal reflectance microscopy (Shortt et al., 2007; Miri et al., 2012; Deng et al., 2012) and optical coherence tomography (OCT) (Bizheva et al., 2011; Lathrop et al., 2012). Shortt et al. (2007) documented the portion of the limbus located toward the cornea using confocal and electron microscopy, and described the presence of both limbal crypts (LCs) between the palisades of Vogt and focal stromal projections (FSPs) at the corneal edge of the limbus, extending in a fingerlike pattern from the palisades and hence appearing circular/oval in the en face imaging plane of confocal microscopy. Dua et al. (2005) and Shanmuganathan et al. (2007) used histology to document the existence of larger, less frequent crypt structures that they termed limbal epithelial crypts (LECs) that contained radial, circumferential and oblique interconnecting components and descended from the epithelium to beneath the scleral surface. Confocal reflectance microscopy, using for example the HRT II with the Rostock module (Heidelberg, Germany) can perform non-invasive imaging of the limbal region, in ex vivo or in vivo tissues (Miri et al., 2012), but its small field size $(300-400 \mu \mathrm{m} \times 300-400 \mu \mathrm{m})$ prevents viewing of the whole of the limbal region in depth in a single acquisition, and crosssectional information is not available. In addition, histology and electron microscopy are invasive and require fixation, staining, and slicing of tissue. OCT imaging, along with confocal microscopy, of the 3D structure of the limbus in fixed human corneoscleral rims (Lathrop et al., 2012) revealed a combination of the structures described in the literature including the variety of palisade and interpalisade patterns with their intra and inter-individual variability, along with structures that may correspond to LCs, LECs and FSPs. The current study sought to assess stem cell presence in relation to morphology by exploring the 3D structure of the limbal crypts using FFOCM, a technique that allows non-invasive micrometric exploration of the 3D volume in unfixed, unsliced ex vivo tissue without staining.

OCT is an imaging modality analogous to ultrasound but using light reflected from structures inside tissue (Huang et al., 1991). Conventional OCT is widely used in ophthalmology to image both the anterior and posterior segment. Axial resolution is typically on the order of $5 \mu \mathrm{m}$, and lateral resolution on the order of $10-20 \mu \mathrm{m}$.

FFOCM (Beaurepaire et al., 1998; Dubois et al., 2002) is a variant of conventional OCT in which two dimensional (2D) en face images are captured on a camera and three-dimensional (3D) data sets may be obtained by scanning in the depth direction. This configuration and the use of a white light source allow for higher axial and transverse resolution than conventional OCT, on the order of $1 \mu \mathrm{m}$. No contrast agents are required as contrast is entirely endogenous. FFOCM can therefore perform micrometer resolution 3D imaging non-invasively in fresh or fixed ex vivo biological tissue samples. FFOCM has been used to image ophthalmic tissues including cornea (Ghouali et al., 2014; Akiba et al., 2007), lens and retina (Grieve et al., 2004), as well as skin (Dalimier and Salomon 2012), brain (Assayag et al., 2013a), breast (Assayag et al., 2013b) and gastrointestinal tissues (Grieve et al., 2014).

Here FFOCM was applied to imaging of the limbal region of the corneoscleral rim of humans, pigs and mice, in order to further understanding of the 3D architecture of the limbal stem cell niche in unfixed tissues in these three species.

\section{Materials and methods}

\subsection{Tissues}

This study was carried out according to the tenets of the Declaration of Helsinki and followed international ethical requirements for human tissues, and was approved by our local institutional review board for human experimentation.

Forty seven human corneoscleral rims were imaged, of which 21 were from males, 18 females, 8 sex unknown, with an average donor age of 71 (range 40-93).

Of the 47 corneoscleral rims imaged, 43 were organ-cultured and 4 fresh, of which 9 ( 7 organ-cultured and 2 fresh) were cultured for colony forming assays (CFA).

The forty three human cadaveric corneoscleral rims from donors originated from the Etablissement Français de Sang (EFS) cornea bank at Saint-Antoine Hospital, Paris, and were retained after the central corneas had been used for keratoplasty procedures on patients (8-mm trephination of the donor tissue). The donor corneas were preserved in CorneaMax (EuroBio, France) medium for a maximum of 35 days at $31{ }^{\circ} \mathrm{C}$, in accordance with European Eye Banking regulations. They were then placed in CorneaJet (EuroBio, France) medium containing Dextran for deturgescence $48 \mathrm{~h}$ prior to transplantation, and transferred to the Quinze-Vingts Ophthalmology Hospital where grafts were performed. The corneoscleral rims were retained in Corneajet (EuroBio, France) medium and imaged within $24 \mathrm{~h}$. Following imaging, they were transferred to the cell culture laboratory.

The four fresh cadaver donor corneas were obtained from the Surgery School of the Assistance Publique - Hôpitaux de Paris (AP$\mathrm{HP}$ ). These were imaged $<24 \mathrm{~h}$ post-mortem, then transferred to the cell culture laboratory.

All animal manipulation was performed in accordance with the ARVO Statement for the Use of Animals in Ophthalmic and Vision Research, and was approved by our local institutional review board for animal experimentation.

Fifteen pig eyes (albino English - pigmented Belgian crossbreed) were obtained from a private research facility. Eyes were removed within one hour after sacrifice of the animal and stored in phosphate buffered solution (PBS). On arrival at the FFOCM imaging location $(<4$ h post-mortem) they were transferred to DMEM medium and dissected for imaging. The corneoscleral button was removed and relief incisions made in the sclera in order to flatten the cornea for imaging. Of the fifteen pig eyes imaged, all were imaged fresh and one placed in organ-culture for 31 days in a flatmount position, and 3(2 fresh, plus the one organ-cultured) were cultured for CFA. 
Twenty-seven mouse eyes (C57 BL/6, Janvier labs, France) were obtained from the animal facility of the Vision Institute. Of the 27 mouse eyes imaged, 21 were fresh, unfixed tissue prepared for the current study, while six were recuperated from an unrelated study and had been fixed using $4 \%$ paraformaldehyde (PFA) and mounted on microscope slides. Mice were deeply anesthetized by pentobarbital and sacrificed by cervical dislocation. After sacrifice, the eyes were immediately removed and dissected to flatmount the cornea for imaging. 8 of the fresh mouse eyes were cultured for CFA. Two of the fresh mouse eyes were organ-cultured for 31 days in a flatmount position, imaged, and then also cultured for CFA.

To resume, 47 human (4 fresh, 43 organ-cultured), 15 pig (15 fresh, of which 1 subsequently organ-cultured) and 27 mouse (22 fresh, of which 2 subsequently organ-cultured, 6 fixed) were imaged; of which 9 human, 3 pig and 10 mouse samples were cultured for CFA.

\section{2. $F F O C M$}

The FFOCM instrument used is a commercial system (LLTech, France). It has been described previously (Ghouali et al., 2014). Illumination is provided by a halogen source, whose short coherence length leads to an axial resolution of $1 \mu \mathrm{m}$. The full field is illuminated and images are captured on a complementary metal oxide semiconductor (CMOS) camera. The interferometer arms hold a matched pair of microscope objectives in the Linnik configuration. $10 \times$ water immersion objectives with 0.3 numerical aperture lead to a lateral resolution of $1.6 \mu \mathrm{m}$. Penetration depth depends on tissue content and transparency and ranges from approximately $200 \mu \mathrm{m}$ in sclera to $1000 \mu \mathrm{m}$ in cornea. Wide field images can be displayed by automated mosaicing to image samples up to $25 \mathrm{~mm}$ in diameter, or stacks of images in depth can be captured. A $2 \times 2 \mathrm{~mm}^{2}$ stitched field is captured in less than five minutes, and a $200 \mu \mathrm{m} \times 1 \mathrm{~mm} \times 1 \mathrm{~mm}$ 3D stack is also captured in less than five minutes.

\subsection{Image acquisition}

For all species, wide field en face FFOCM images of the whole of each corneoscleral rim were captured along with 3D stacks of images at four equally spaced sampling points around each corneoscleral rim. Each of these stacks could be navigated in multiplanar, video or 3D mode in order to view the crypt structure. A photograph of each corneoscleral rim was also saved.

The wide field stitched image of each corneoscleral rim was captured close to the surface at a depth where the palisades of Vogt were most clearly visible (Fig. 1A). The four wide field image stacks (of depth $\times$ width $\times$ length of $150 \mu \mathrm{m} \times 2 \mathrm{~mm} \times 4 \mathrm{~mm}$ in human, $200 \mu \mathrm{m} \times 2 \mathrm{~mm} \times 4 \mathrm{~mm}$ in pig, and $150 \mu \mathrm{m} \times 800 \mu \mathrm{m} \times 800 \mu \mathrm{m}$ in mouse) were then captured at top, bottom, left and right regions of the corneoscleral rim (Fig. 1A-E), to provide the most complete coverage possible of the limbal region in a reasonable timeframe that yielded manageable data sets.

An incision was made in the sclera to mark $0^{\circ}$ for the imaging data, and on transfer to the cell culture laboratory, the corneoscleral rim was cut into eight $45^{\circ}$ segments for human and pig, numbered one to eight in a clockwise fashion. Each image data volume straddled two of the segments, so providing a sampling of crypt volume in each cultured explant. The protocol of donor cornea collection for the French Eye Banks and the Surgery School does not include recording information on the orientation of the cornea. In order to determine vertical (superior/inferior) and horizontal (temporal/nasal) axes, the long and short axes of the anterior surface of each cornea were measured on the photographs. Crypt distribution around the $360^{\circ}$ circumference of the cornea could therefore be related to location on the vertical (superior/inferior) or horizontal (temporal/nasal) axes.

In mouse, each whole cornea was divided into four explants, each correlated to an image stack. Eye orientation was not recorded.

\subsection{Image analysis}

\subsubsection{Qualitative assessment of crypt-richness of entire corneoscleral rim}

The wide-field FFOCM images (Fig. 1A) at a single depth typically $30 \mu \mathrm{m}$ below the surface in organ-cultured human samples, $50 \mu \mathrm{m}$ in fresh human samples (where intact, thick epithelium meant the palisades of Vogt were located deeper than in organ-cultured samples), $70 \mu \mathrm{m}$ in pigs and $10 \mu \mathrm{m}$ in mouse were assessed to qualitatively determine overall visibility of limbal crypt structures and the palisades of Vogt in each corneoscleral rim, and also to identify potential crypt-rich areas within each corneoscleral rim. The wide-field image depth tended to lie toward the surface of the palisades of Vogt and the interpalisade epithelial crypts, where the typical limbal palisade patterns were most easily recognized, while sub-scleral crypts tended to be deeper. Morphological crypt features including shape (rectangular or rounded) and orientations (radial or circumferential) were tallied for all samples to give a percentage of overall number of samples with a particular feature visible.

\subsubsection{Limbal crypt volume}

Image processing software (Fiji, National Institutes of Health, USA) was used to perform thresholding and area quantification on slices averaged in $10 \mu \mathrm{m}$ depth bundles in each 3D image stack. Volumes were expressed as the percentage of each stack occupied by crypts:

$\% V=100 \times\left(\sum S A \times 10\right) / v$

where $S A=$ surface area occupied by crypts in each $10 \mu \mathrm{m}$ en face bundle, over volume $v=$ depth $\times$ width $\times$ length of $150 \mu \mathrm{m} \times 2 \mathrm{~mm} \times 4 \mathrm{~mm}$ in human, $200 \mu \mathrm{m} \times 2 \mathrm{~mm} \times 4 \mathrm{~mm}$ in pig, and $150 \mu \mathrm{m} \times 800 \mu \mathrm{m} \times 800 \mu \mathrm{m}$ in mouse.

\subsection{Fluorescent labeling of epithelial and stem cells}

As the contrast mechanism in FFOCM is intrinsic, due to changes in refractive index, fluorescent labels are not detected in FFOCM. In order to demonstrate the presence of stem cells and epithelial cells in the limbal crypt architecture imaged by FFOCM, we labeled and imaged specimens using fluorescence microscopy. We used both an Olympus laser scanning microscope FV-1200 (Olympus France, Rungis) for confocal fluorescence imaging of labeled specimens, with overlay of the fluorescence and FFOCM images in postprocessing, and a prototype multimodal optical microscope that allows simultaneous co-located FFOCM and fluorescence imaging. Compared to previous fluorescence-FFOCM setups (Harms et al., 2012; Auksorius et al., 2012), in the set-up used here, fluorescence is acquired simultaneously with the FFOCM measurements, meaning image capture is faster.

Briefly, this set-up operated in a similar fashion to conventional FFOCM but with the addition of a blue light emitting diode (LED) (Thorlabs, M470L2, $650 \mathrm{~mW}$ ) centered at $470 \mathrm{~nm}$ blocked by an excitation filter $(\lambda=500 \mathrm{~nm}$, Thorlabs, FES0500) to excite fluorophores. The two illumination beams are combined and focused onto the sample, and in the detection path, FFOCM and fluorescence signals are separated by a Single Edge Dichroic Beamsplitter 


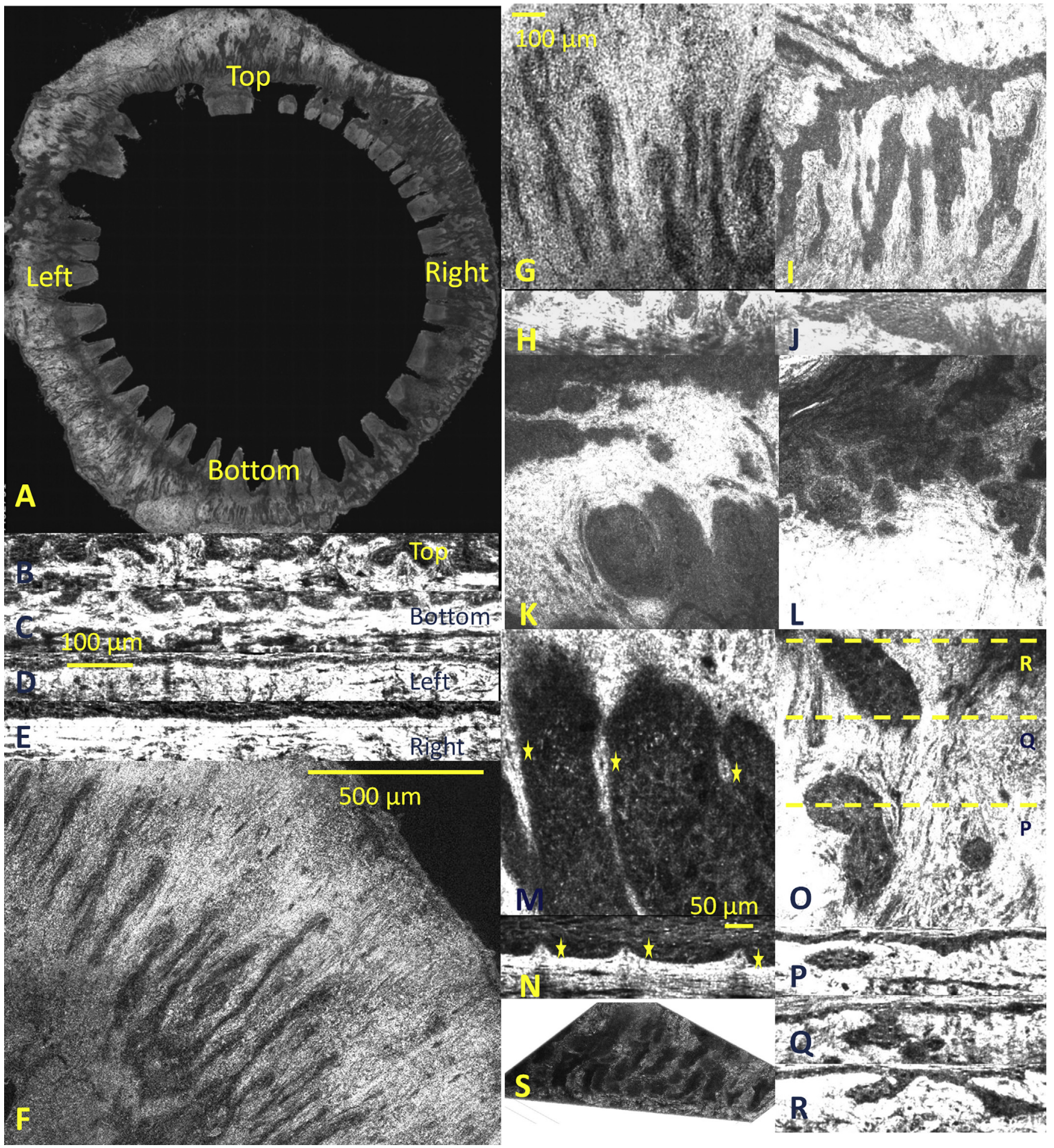

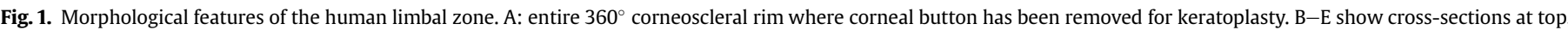

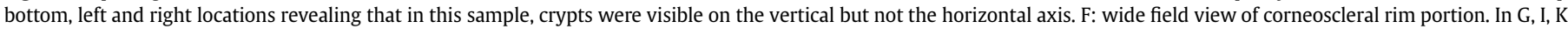

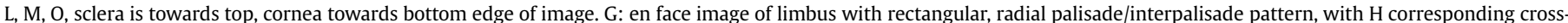

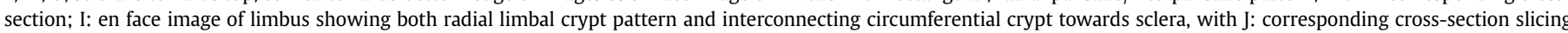

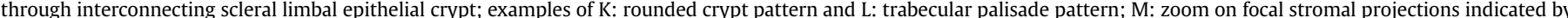

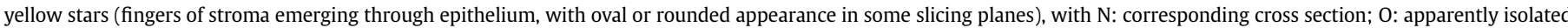

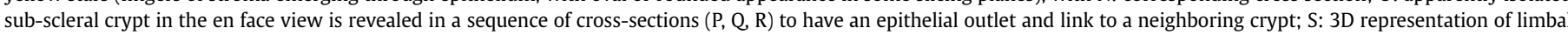
zone. Supplementary movies 1 and 2 show en face and cross-sectional fly-throughs respectively corresponding to I, J here.

(BrightLine, $\lambda=593 \mathrm{~nm}$, Di02-R594-22x27), and captured by two cameras, a CMOS from PhotonFocus for the FFOCM image and a scientific CMOS from PCO.edge to capture the fluorescence image, with additional filters placed in front of the detection cameras to ensure independence of the two paths. As the optical path into the tissue is parallel for the FFOCM and fluorescence channels, an 
identical region of the sample is captured simultaneously on the FFOCM and fluorescence cameras. 30×, NA 1.05 UPLSAPO oil immersion microscope objectives (Olympus France, Rungis) produced a field size of $260 \mu \mathrm{m} \times 260 \mu \mathrm{m}$ and a pixel sampling of $255 \mathrm{~nm}$ per pixel. FFOCM image capture time is $2 \mathrm{~ms}$, at a frame rate of 100 images per second. The fluorescence channel displays only one image, exposed for $800 \mathrm{~ms}$.

Corneoscleral rims were first imaged using the LLTech FFOCM system in order to have a wide-field image for correlation with the fluorescence images that cover smaller zones. Corneoscleral rims were then divided into three segments and progenitor cells were identified with a whole mount immunofluorescence assay. Briefly, after fixation with $4 \%$ PFA for $1 \mathrm{~h}$, the cells were permeabilized and blocked with $0.5 \%$ triton X-100 and $0.2 \%$ gelatin in PBS for one night at room temperature followed by incubation with the anti cytokeratin 3 antibody (1:100) (monoclonal antibody Clone AE-5; Dako Trappes, France) and anti p63- $\alpha$ polyclonal antibody (1:50) (cell signaling technology, France) for $24 \mathrm{~h}$ at room temperature, and then with the secondary antibody (Alexa Fluor 488,1:600) for $2 \mathrm{~h}$ at room temperature, and nuclei were counterstained with DAPI. One segment was incubated with phosphate-buffered solution (PBS) instead of the primary antibody to serve as a negative control.

Fluorescence-FFOCM images were taken of all three segments using the multimodal system. As the fluorescence-FFOCM set-up used allows imaging in only the green wavelength band, the Olympus confocal fluorescence microscope was preferred when we wished to label several cell types at once.

\subsection{Colony forming assays}

After FFOCM analysis, 9 human corneoscleral rims (2 fresh, 7 organ-cultured) were divided into 8 explants per rim, 3 pig corneoscleral rims ( 2 fresh, 1 organ-cultured) into 8 explants per rim, and 10 mouse whole corneas ( 8 fresh, 2 organ-cultured) into 4 explants per cornea. According to the protocol descried in Ghoubay-Benallaoua et al., 2013, explants were digested using $1 \mathrm{mg} / \mathrm{ml}$ collagenase A (Roche Diagnostics, Mannheim, Germany) in cholera toxin-free Green medium at $37^{\circ} \mathrm{C}$ for $18 \mathrm{~h}$. Limbal epithelial cells were collected from the incubated tissues and dissociated into single cells by pipetting. The clonal growth ability of cultured limbal epithelial cells was evaluated by determining CFE. Swiss albino murine 3T3 fibroblasts (ATCC, Molsheim, France) were treated with $4 \mu \mathrm{g} / \mathrm{ml}$ mitomycin $\mathrm{C}$ for $2 \mathrm{~h}$ and then trypsinized and plated at a density of $2 \times 10^{4} / \mathrm{cm}^{2}$ onto six-well culture plates. Enzymatically dissociated cells were seeded at low density ( 1000 cells/well) in six-well culture plates on 3T3 fibroblasts feeder layers (Nakamura et al., 2004). Cultures were incubated at $37{ }^{\circ} \mathrm{C}$ under $5 \% \mathrm{CO}_{2}$. They were observed three times a week by inverted phase-contrast microscopy. Growing epithelial cells were easily differentiated from feeder cells by morphology (i.e., polygonal versus spindle-shaped cells). A colony was defined as a group of eight or more contiguous adherent epithelial cells as described elsewhere (Ang et al., 2004; Nakamura et al., 2006; Yokoo et al., 2008). Given the low density of seeded epithelial cells, colonies were not contiguous. The epithelial colonies were fixed on day 12 and stained with crystal violet and photographed. The CFE was defined as the percentage of cells forming colonies as follows: CFE $(\%)=($ number of counted colonies/number of seeded cells $) \times 100$. As explants in human and pig corneoscleral rims covered the entire limbal region, and in the mouse covered the entire cornea, the epithelial colonies formed could have originated from any location within these regions, i.e. we could not discriminate between clones originating from the corneal side of the limbus, scleral side of the limbus, or in mouse, central cornea.

\subsection{Statistical analysis}

One-way ANOVA was used to compare continuous variables between more than 2 groups. The Brown-Forsythe test was used to examine homogeneity of variance between groups. Where a significant difference between groups was detected, a post-hoc analysis was performed with the Tukey post-hoc test where group variance was homogenous and the Games-Howell post-hoc test where group variance was not homogenous. Wilcoxon rank sum test was used to compare continuous variables between 2 groups. Spearman rank correlation coefficient was used to assess correlation between continuous variables.

\section{Results}

\subsection{Human}

\subsubsection{Description of crypt morphology}

3.1.1.1. Organ-cultured corneas. Limbal crypts were observed in $90 \%$ of the organ-cultured human corneoscleral rims. Morphological description tallies indicated that crypt pattern including shape and orientation was highly variable from one individual to another and from one region of the limbus to another in the same subject. In en face views, rectangular shaped, radially oriented interpalisade crypts were seen in $74 \%$ of eyes (Fig. $1 \mathrm{~F}-\mathrm{J}$ ), rounded interpalisade crypts in $23 \%$ of eyes (Fig. $1 \mathrm{~K}, \mathrm{~L}$ ), with interconnecting circumferential crypts in 56\% of eyes (Fig. 1I, J). Multiple crypt forms and orientations were seen in the same eye in $75 \%$ of eyes. Between the palisades of Vogt, crypts rose to the surface at the cornea, corresponding to LCs (interpalisades). Palisades often terminated in FSPs (Fig. 1M, N). On moving outward to the sclera, crypts then invaginated beneath the scleral surface (Fig. 10-R) corresponding to LECs. In cross-sectional views, crypts appeared toward the corneal edge as simple valleys between the palisades of Vogt (seen in $79 \%$ of eyes), which on moving toward the sclera became enlarged with a narrower epithelial outlet (seen in $41 \%$ of eyes), and then invaginated to a cavern beneath a scleral roof at the scleral side of the limbus (seen in $40 \%$ of eyes) (Fig. $1 \mathrm{H}, \mathrm{J}$, $\mathrm{P}, \mathrm{Q}, \mathrm{R}$, Supplementary movie 2 ). Sub-scleral caverns could usually be followed to their epithelial outlet (Fig. $1 \mathrm{P}-\mathrm{R}$ ). On occasions where these sub-scleral caverns appeared to be isolated in surrounding sclera, their path to the epithelium may have passed outside of the imaged zone.

Supplementary video related to this article can be found at http://dx.doi.org/10.1016/j.exer.2015.08.003.

3.1.1.2. Fresh corneas. Little difference was observed in the crypt morphology between fresh and organ cultured specimens, aside from epithelial degradation. Fresh cadaver corneas had similar morphological features though had a greater quantity of intact epithelium, so maintaining a thicker epithelial roof with visible cellular details (Fig. 2, Supplementary movie 3). Indeed, under superficial and wing cells layers, small, highly scattering basal cells with large nuclear to cytoplasmic ratio could be identified clinging to palisade walls and in sub-scleral caverns.

\subsubsection{Analysis of crypt distribution, area and volume around the limbus}

Crypt distribution around the $360^{\circ}$ circumference of the cornea could be related to location on the vertical (superior/inferior) or horizontal (temporal/nasal) axes by measuring the short (vertical) and long (horizontal) axes in each cornea (Fig. 1A-E).

Crypts were detected on two opposing sides in $31 \%$ of eyes, on two neighboring sides in $18 \%$ of eyes, on 3 or 4 sides in $10 \%$ of eyes and on only one side in $41 \%$. Average angular spread of crypt 


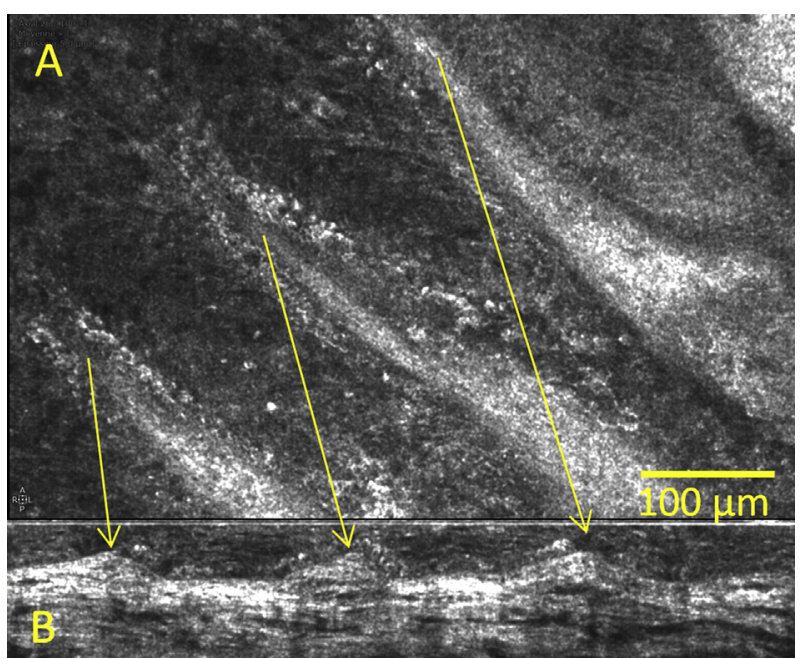

Fig. 2. Fresh human cornea. More intact epithelium can be noted than in organcultured cornea of Fig. 1, with epithelial cell details, particularly small, highly scattering basal cells clinging to palisade walls. A: en face view of palisades, with B: crosssectional view of this zone. See supplementary material movie 3 to view en face movie descending in depth from surface to bottom of crypt structures.

clusters was $50^{\circ}$ (range $15^{\circ}-120^{\circ}$ ) with an average of 2 clusters per cornea (range 1-4) for all corneas with visible crypts. Average crypt depth in humans was $50 \mu \mathrm{m}$ (range $35 \mu \mathrm{m}-120 \mu \mathrm{m}$ ) and width $50 \mu \mathrm{m}$ (range $15 \mu \mathrm{m}-150 \mu \mathrm{m}$ ).

Limbal crypt volume per quadrant represented on average $13.0 \%$ (range $0-62 \%$ ) of the limbal volume sampled ( $2 \mathrm{~mm} \times 4 \mathrm{~mm} \times 150 \mu \mathrm{m})$. This figure was $15.8 \%$ for the vertical location and $8.1 \%$ for the horizontal location $(p=0.007)$. The $95 \%$ confidence interval of the limbal crypt volume per cornea was $0.0 \%-29.9 \%$.

$\% V$ was significantly greater $(\mathrm{p}<0.0001)$ in fresh $(39.8 \%$, range $23 \%-56 \%$ ) than in organ-cultured tissue.

No correlation of $\% V$ with age or age group (in 10 year groups) was observed in this study ( $\mathrm{p}=0.29)$.

\subsubsection{Fluorescent labeling of cells}

Fluorescent labeling confirmed presence of p63- $\alpha+$ stem cells and epithelial cells with corneal differentiation (i.e., cytokeratin $3+$ cells) in the crypt architecture (Fig. 3). These are seen to be coincident with the crypt structures viewed with FFOCM (Fig. 3A-D). Controls were negative (Fig. 3E, F). Imaging with confocal fluorescence microscopy allowed merged viewing of stem cells with other cell populations, showing that both stem and epithelial cells are present in human limbal crypts (Fig. 3G, H), though not colocalized.

\subsubsection{Correlation between morphology and clone production}

Colonies grown from dissociated limbal epithelial cells were associated with areas visibly rich in crypts (Fig. 4). The CFE(\%) significantly increased with limbal crypt volume (\%), per segment $\left(r_{\mathrm{s}}=0.42, \mathrm{p}=0.001\right)$ and per cornea $\left(\mathrm{r}_{\mathrm{s}}=0.82, \mathrm{p}=0.02 ;\right.$ Fig. $\left.4 \mathrm{~B}\right)$. It should be noted that large $\% \mathrm{~V}$ over the sampled area does not necessarily mean large crypts: large $\% V$ can equally mean numerous small crypts.

The average CFE(\%) was $1.8 \%$ overall, of which $2.4 \%$ was on the vertical axis and $1.4 \%$ on the horizontal axis ( $\mathrm{p}=0.17)$.

In fresh cadaver corneas, CFE(\%) was $2.1 \%$ overall, which was not significantly greater than in organ-cultured cornea $(\mathrm{p}=0.67$; Fig. 4D).

\subsection{Pig}

In pig eyes (Fig. 5B), the palisade zone was located further toward the scleral than the corneal side of the limbus. Palisades were fine and interpalisade crypts wide and oval, in a uniform radial orientation, connecting to numerous narrow invaginations beneath the sclera in a trabecular pattern. Distribution was relatively uniform and continuous around the $360^{\circ}$ of the eye as well as having similar depth $(50 \mu \mathrm{m})$, width $(90 \mu \mathrm{m})$, shape (oval) and orientation (radial) in all eyes. The limbal zone was $1.5 \mathrm{~mm}$ wide.

Limbal crypt volume represented on average 9.5\% (range $3-21 \%)$ of the limbal volume sampled $(200 \mu \mathrm{m} \times 2 \mathrm{~mm} \times 4 \mathrm{~mm})$. This figure was $9.2 \%$ for the vertical location and $9.8 \%$ for the horizontal location $(\mathrm{p}=0.67)$.

Fluorescent labeling revealed p63- $\alpha+$ cells contained within crypts in pig (Fig. 3I, J). CFE(\%) in fresh pig samples was $0.59 \%$ overall, of which $0.45 \%$ was on the vertical axis and $0.70 \%$ on the horizontal axis $(\mathrm{p}=0.17)$.

Organ-cultured pig cornea, after 31 days in organ culture conditions, showed epithelial degradation including detachment and undulations that increased surface scattering from the tissue, thereby decreasing penetration depth of the FFOCM images. The limbal crypt structure was faintly visible beneath the damaged epithelium and the palisade/interpalisade pattern appeared intact, though quantification was not possible due to the degraded image quality. CFE(\%) in organ-cultured pig cornea was zero. This was not significantly different from CFE(\%) in fresh pig cornea (Fig. 4D).

\subsection{Mouse}

In fresh mouse cornea, the palisades of Vogt were not observed and the limbal region contained only an epithelial trough circumferentially surrounding the cornea (Fig. 5C). \% $\mathrm{V}$ was therefore evaluated at zero for all mouse samples. Architecture of the limbus appeared unchanged after 31 days in organ culture, though the epithelium had thinned and surface cells were misshapen.

Fluorescent labeling with DAPI and p63- $\alpha$ using confocal fluorescence microscopy showed clumps of p63- $\alpha+$ cells present in the limbal trough, along with fewer isolated p63- $\alpha+$ cells distributed throughout the central cornea (Fig. 3K, L, M). CFA was unsuccessful in both fresh and organ-cultured mouse cornea, yielding a CFE(\%) of zero for all samples.

\subsection{Interspecies variability}

Morphology of the limbal stem cell niche differed between species (Fig. 5).

CFE(\%) according to tissue characteristics is shown in Fig. 4D. It was significantly higher in human tissues (either fresh or organcultured) than in pig and mouse tissues ( $p<0.01)$. Differences between pig and mouse tissues were not significant $(p>0.2)$. For all 3 species there were no significant differences in $\mathrm{CFE}(\%)$ between fresh and organ-cultured tissues $(\mathrm{p}>0.3)$.

\section{Discussion}

\subsection{Crypt architecture}

Crypt structures were detected in $90 \%$ of the human samples examined. This is in agreement with the reported value of visibility of the palisades of Vogt in 80-90\% of subjects (Townsend, 1991; Patel et al., 2006; Dua et al., 2006). Visibility of palisades has been shown to be lower in non-pigmented individuals (Miri et al., 2012) and to decrease with age (Goldberg and Bron, 1982). The corneoscleral rims we obtained had an average age of 71 (range 


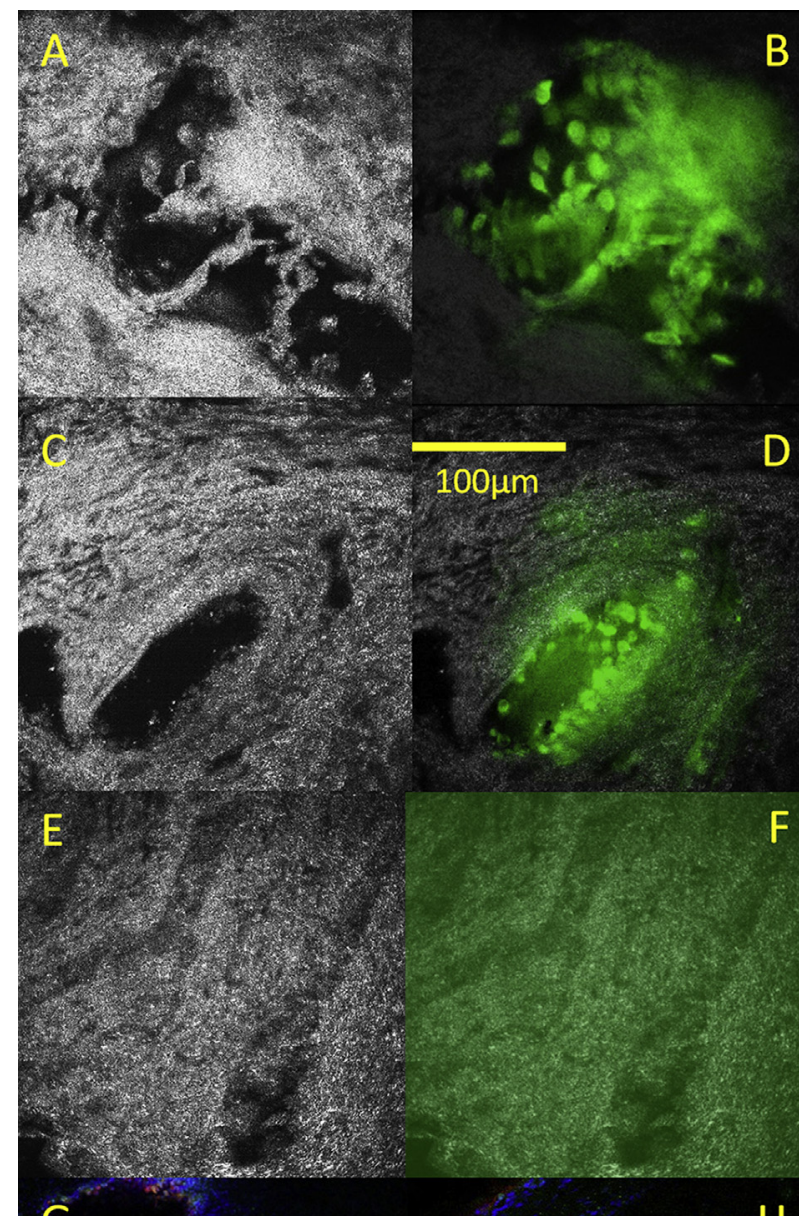

G
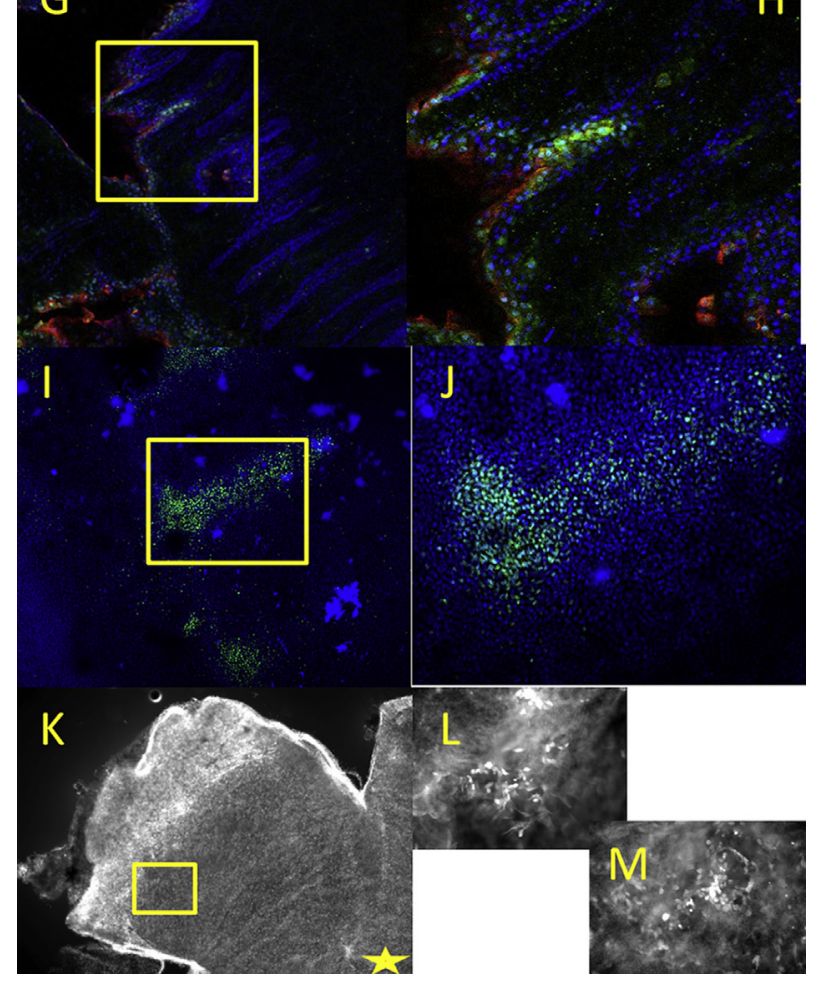

Fig. 3. Fluorescent labeling of stem cells. A, C, E: FFOCM images of crypts $(260 \mu \mathrm{m} \times 260 \mu \mathrm{m}$ field size) from the fluorescence-FFOCM setup with the fluorescence channel switched off. B, D, F: With the simultaneous fluorescence imaging, the fluorescence-FFOCM combined images show presence of epithelial cells (in green, B)
40-93), and although information on each individual's racial origin was not supplied with the donor corneas, racial origin of donors is predominantly European-Caucausian (non-pigmented) in the French Eye Bank network. This may explain why palisades were not detected in $10 \%$ of samples imaged.

While Goldberg and Bron (1982) and Townsend (1991) used slit lamp examination to describe the morphology of the limbal region as containing a variety of palisade and interpalisade patterns and forms, more precise microscopic morphology described by Shortt et al. (2007) included the presence of both LCs between the palisades of Vogt and FSPs at the corneal edge of the limbus, while Dua et al. (2005) and Shanmuganathan et al. (2007) describe the existence of larger, less frequent crypt structures that they term LECs that contained radial, circumferential and oblique components and descended beneath the scleral surface. In the current study, all of the features previously described were seen. FSPs were less frequently detected as distinct features than in previous studies (Shortt et al., 2007; Miri et al., 2012), possibly due to epithelial degradation in organ-cultured cornea making them appear simply as terminations of the palisades. Miri et al., 2012 used laser scanning confocal microscopy to image the limbal zone in vivo, in depth in 46 subjects. They describe a similar crypt architecture to that detected here on the surface, however in depth they suggest that palisades and interpalisade crypts (or epithelial rete pegs) inverse their contrast, so that the structures which interlink the network beneath the conjunctival surface are identified as the palisades. Via 3D navigation of our data sets to the depths at which this contrast inversion was observed, we suggest that the dark regions below palisades are caused by shadowing from the palisades on the surface, and at this depth we are in fact viewing only sclera. We identified interlinking dark structures towards the conjunctiva, at depths between $0 \mu \mathrm{m}$ and $120 \mu \mathrm{m}$, as crypts rather than palisades.

While Goldberg and Bron 1982 and Shortt et al., 2007 found that crypt rich areas were most commonly located in superior and inferior regions, where the cells would be best protected by the eyelids, Shanmuganathan et al., 2007 found the highest incidence of crypts in a temporal location, followed by an equal frequency superiorly and inferiorly, with least frequent incidence nasally. The current study discovered superior or inferior crypt locations to be double the incidence of a temporal or nasal location. It would be desirable to relate our data on $360^{\circ}$ distribution of crypts to temporal/nasal/superior/inferior quadrants, rather than just the axis. This could be achieved by creating a specific protocol for donor cornea removal and storage of the Eye Banking network of France to include orientation information.

The precise non-invasive 3D imaging performed using FFOCM provides information on the morphology of the limbal crypt network on unfixed, unstained, whole tissue samples. This morphological information could be valuable in the design of artificial molds for stem cell culture for artificial limbus fabrication (Levis et al., 2013). Our quantitative assessment of the limbal crypts

and stem cells (in green, D) in the crypt architecture. Controls (E, F) showed only a uniform noise background in the fluorescence channel (F). G, H: human limbus imaged with a confocal fluorescence microscope with an X10 objective $(G)$ and zoom $(H)$ showing p63- $\alpha+$ (green), cytokeratin $3+$ (red) and DAPI (blue) staining, where the sclera lies to the left and the cornea to the right of the image. p63- $\alpha+$ cells are visible in the base of a few crypts, while cytokeratin 3 + cells are not colocalized with p63- $\alpha+$, and are present in greatest number in higher imaging planes toward the sample surface and toward the cornea. I, J: porcine cornea imaged with a confocal fluorescence microscope with an X10 objective (I) and a X20 objective (J) showing p63- $\alpha+$ (green), and DAPI (blue) staining, where the sclera lies to the left and the cornea to the right of the image. p63- $\alpha+$ cells are visible within crypts. K: portion of a flat mounted mouse cornea at $5 \times$, marked with p63- $\alpha$ and DAPI, showing location of central cornea (yellow star) and sclera, L: zoom on limbal region at $20 \times$ shows clumps of bright p63- $\alpha+$ cells, and $\mathrm{M}$ : at $20 \times$ some p63- $\alpha+$ cells distributed throughout central cornea. 


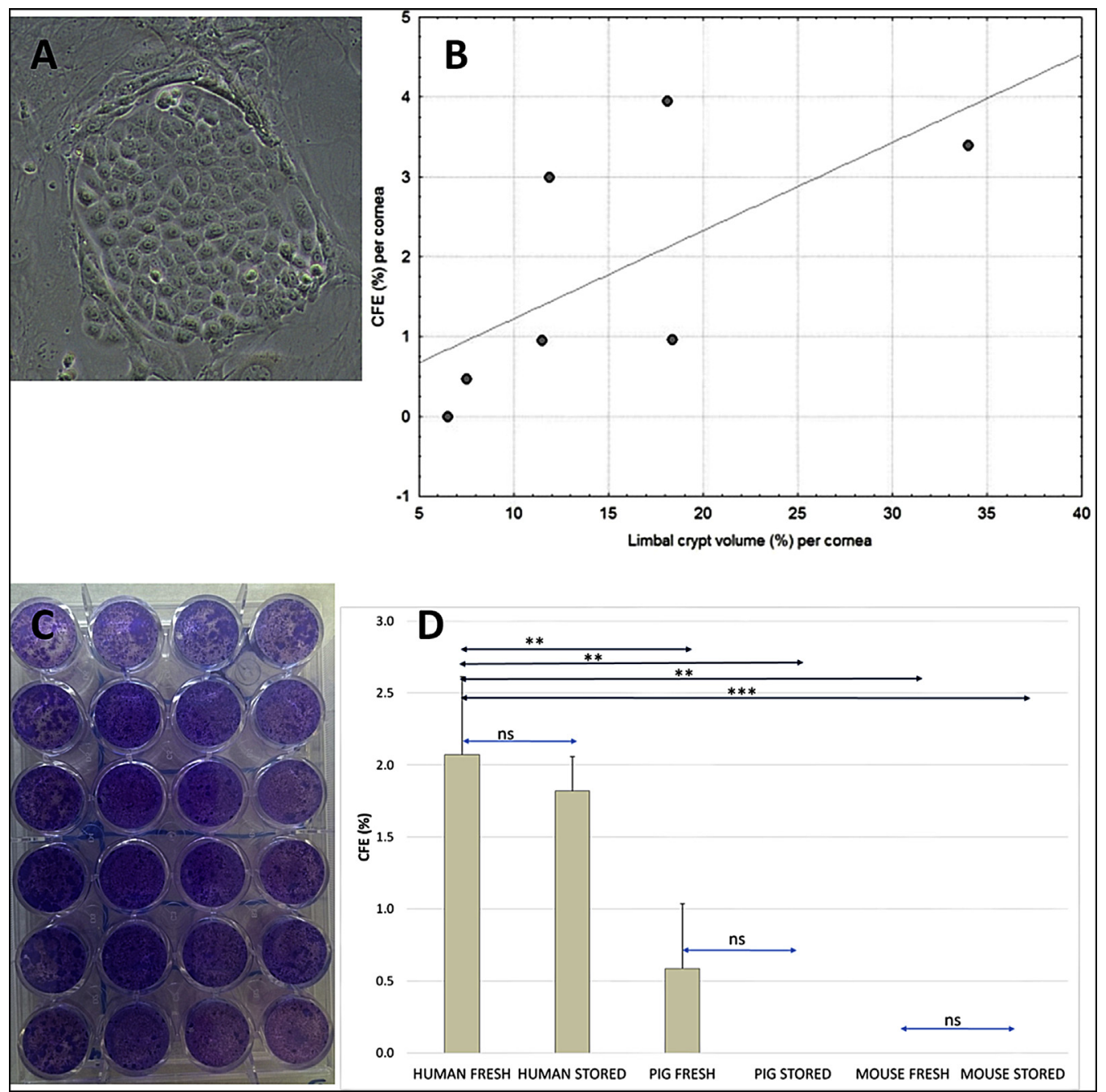

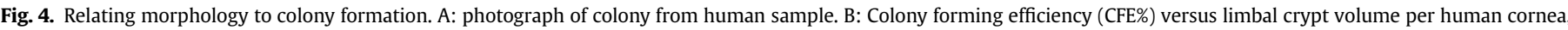

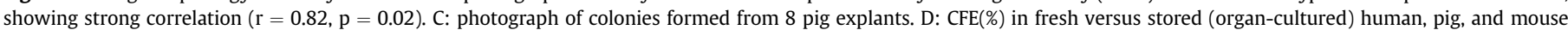

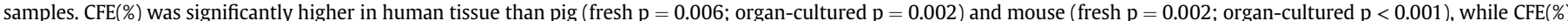

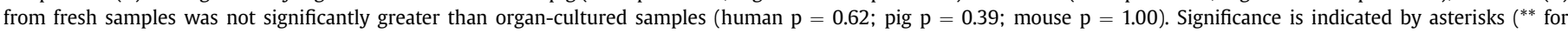
$0.01>\mathrm{p}>0.001$; $^{* * *}$ for $\mathrm{p}<0.001$, ns for not significant).

showed that stroma represented the most important component of the niche volume with the epithelial component representing less than $20 \%$

The data on pig and mouse crypt architecture indicate the differences between species. Radially oriented crypts extending beneath the scleral roof were found in humans and pigs but not in mice. This architecture provides protection from visible light and ultraviolet irradiation. Interestingly, the former species have a diurnal activity whereas the latter has a nocturnal activity. Ultraviolet (UV) B radiation induces damage to the human corneal

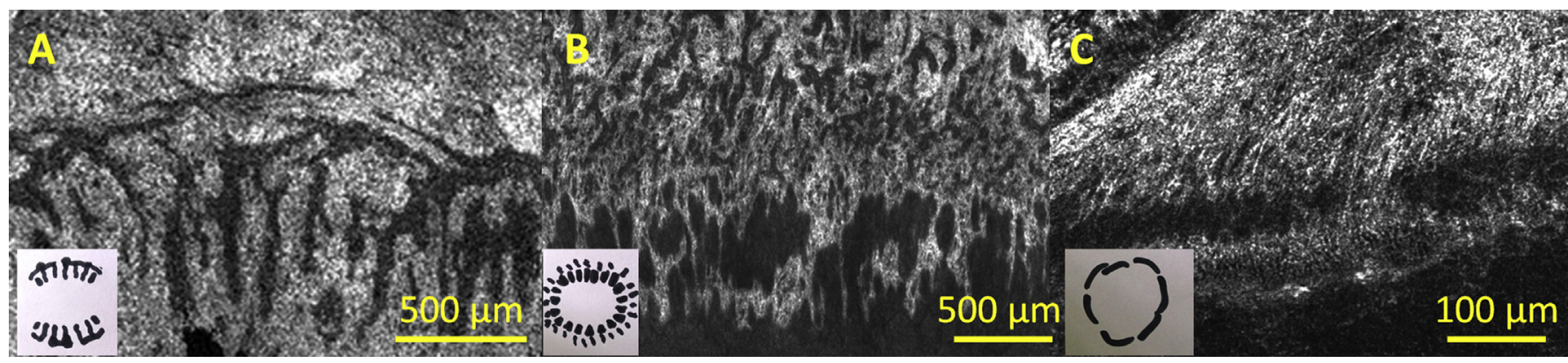

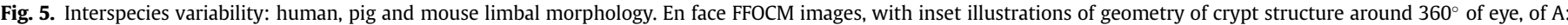

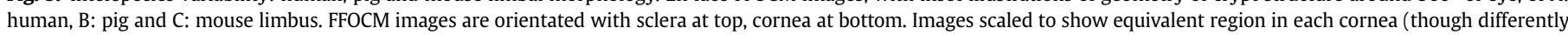
sized due to different eye sizes). 
epithelium, such as epithelial sloughing, decreased mitotic rate, and decreased Langerhans cell density (Borderie et al., 1996; Haaskjold et al., 1993). Interestingly, the porcine limbus has been shown to share features with the human limbus such as high CFE achieved with epithelial cells harvested from crypt regions and expression of the putative stem cell markers p63- $\alpha$ and integrin $\beta 1$ (Notara et al., 2011). The absence of regional variation of crypt volume in pigs as compared to humans could be explained by the different orientation of the eyes with respect to the face. The exposure of pig eyes due to their position on the side of the head and the lack of brow may mean that there is less variation in UV and visible radiation over the $360^{\circ}$ of the eye. In the nocturnal mouse, protection from light has less importance, and this may be the reason that the stem cells are distributed in a limbal trough close to the epithelial surface as well as distributed throughout the central cornea (Majo et al., 2008). Unsuccessful CFA in mouse by the method proposed for human and porcine samples is consistent with the literature (Majo et al., 2008).

\subsection{Fresh versus organ-cultured samples}

Organ culture of human donor corneas is known to induce epithelial sloughing. After the deswelling period in dextransupplemented medium, the corneal epithelium is reduced to two cell layers (Borderie et al., 1995). The remaining epithelial cells appear flattened. They feature normal endoplasmic reticula, glycogen, mitochondria and nuclei, presence of hemidesmosomes on the basal cell membrane of the basal epithelial cells and desmosomes in the epithelium. The epithelial basement membrane is normal (Borderie et al., 1997). In the present study, \%V was reduced in organ-cultured tissue but CFE was preserved. Reduction in crypt volume in organ-cultured corneas may be explained by the reduced thickness of the limbal epithelium in organ-cultured tissue, while the limbal epithelial progenitors, located in the bottom of crypts, are preserved, thereby maintaining CFE.

\subsection{Correlation of fluorescent labeling and FFOCM images}

Use of an FFOCM system coupled with a fluorescence channel, that allows coincident and simultaneous OCT and fluorescence images to be acquired, successfully demonstrated presence of stem cells and epithelial cells within the crypt structures detected by FFOCM. Limitations of the multimodal fluorescence-FFOCM system used included lack of depth sectioning and use of a single color wavelength band for excitation and detection, plus low sensitivity in the fluorescence channel, so that the fluorescent signal was often weak in comparison to images obtained with the Olympus confocal fluorescence microscope. For these reasons, we display images from both fluorescence-FFOCM and confocal fluorescence microscopy. Determining the depth of the stem cells could be achieved by adding sectioning capability to the fluorescence-FFOCM system, and merged images using multiple markers at different emission wavelengths could be achieved by adding multiple illumination paths. A system including these upgrades is currently being developed.

\subsection{Targeted biopsy}

Imaging using FFOCM can provide a quick assessment of whether a corneoscleral rim is rich in crypts, and therefore a good candidate for assessing clone production in culture. This assessment step could improve efficiency in the culture of artificial limbus, by determining optimum samples for CFA. This type of targeted biopsy was previously proposed by Shortt et al., 2007 using confocal reflectance microscopy. However we propose that
FFOCM can provide such information more quickly and easily. Screening of donor corneas in tissue banks to choose the best candidates for limbal graft could also be performed with FFOCM. Proposed future developments of FFOCM technology include use of a camera allowing far more rapid image acquisition, so improving the efficiency of the technique for ex vivo imaging, but also potentially permitting in vivo imaging in the future (Grieve et al., 2005).

\section{Conclusion}

The architecture of the human corneal limbal region has been explored with FFOCM and appears to show that the crypt structure includes narrow, frequent radial components stretching towards the cornea, between the palisades of Vogt, termed LCs by Shortt et al., 2007, as well as larger, wider crypt volumes located toward and extending beneath the sclera in radial, circumferential and oblique directions, termed LECs by Dua et al., 2005 and Shanmuganathan et al., 2007. Crypts appear interlinked below the conjunctival surface at depths around $80 \mu \mathrm{m}$. All crypt-related architectural features found in the current study were contained within a $1 \mathrm{~mm}$ width from cornea to sclera, and $120 \mu \mathrm{m}$ depth. Crypts were more likely to be found on the vertical axis than the horizontal axis in human. Limbal architecture in pig resembled that of humans but featured more homogeneous distribution around the $360^{\circ}$ of the eye, while mouse limbus contained only a circumferential trough without radial crypts visible. Fluorescent labeling confirmed presence of stem cells in the crypt architecture detected. The architecture of the LSC niche appears to be linked to its density in LSC and species behavior and eye position, i.e. diurnal or nocturnal, and front versus side of the head along with presence or absence of brows. Finally, clone production correlated strongly with the limbal crypt volume in humans indicating that limbal crypts constitute a niche for adult limbal stem cells. FFOCM imaging could assist in assessment of the richness of the limbal crypts for targeted biopsy for cell culture in the fabrication of artificial bioengineered cornea.

\section{Acknowledgments}

This project was financed by the Agence Nationale de Recherche (ANR), under a PRTS (Projet de Recherche Translationelle en Santé) grant (ANR-13-PRTS-0009).

\section{References}

Akiba, M., Maeda, N., Yumikake, K., et al., 2007. Ultrahigh-resolution imaging of human donor cornea using full-field optical coherence tomography. J. Biomed. Opt. 12, 041202. http://dx.doi.org/10.1117/1.2764461.

Ang, L.P., Tan, D.T., Beuerman, R.W., et al., 2004. Development of a conjunctival epithelial equivalent with improved proliferative properties using a multistep serum-free culture system. Invest. Ophthalmol. Vis. Sci. 45, 1789-1795. http:// dx.doi.org/10.1167/iovs.03-1361.

Assayag, O., Grieve, K., Devaux, B., et al., 2013a. Imaging of non tumorous and tumorous human brain tissue with full-field optical coherence tomography. Neuroimage Clin. 2, 549-557. http://dx.doi.org/10.1016/j.nicl.2013.04.005.

Assayag, O., Antoine, M., Sigal-Zafrani, B., et al., 2013b. Large field, high resolution full field optical coherence tomography: a pre-clinical study of human breast tissue and cancer assessment. Technol. Cancer Res. Treat. Exp. 1, 21-33. http:// dx.doi.org/10.7785/tcrtexpress.2013.600254.

Auksorius, E., Bromberg, Y., Motiejunaite, R., et al., 2012. Dual-modality fluorescence and full-field optical coherence microscopy for biomedical imaging applications. Biomed. Opt. Exp. 3 (3), 661-667. http://dx.doi.org/10.1364/ BOE.3.000661.

Beaurepaire, E., Boccara, C., Lebec, M., et al., 1998. Full-field optical coherence microscopy. Opt. Lett. 23 (4), 244-246. http://dx.doi.org/10.1364/OL.23.000244.

Bizheva, K., Hutchings, N., Sorbara, L., et al., 2011. In vivo volumetric imaging of the human corneo-scleral limbus with spectral domain OCT. Biomed Opt. Exp. 2 (7), 1794-1802. http://dx.doi.org/10.1364/BOE.2.001794.

Borderie, V.M., Kantelip, B.M., Delbosc, B.Y., et al., 1995. Morphology, histology, and 
ultrastructure of human C31 organ-cultured corneas. Cornea 14, 300-310.

Borderie, V.M., Kantelip, B.M., Genin, P.O., et al., 1996. Modulation of HLA-DR and CD1a expression on human cornea with low-dose UVB irradiation. Curr. Eye Res. 15, 669-679. http://dx.doi.org/10.3109/02713689609008908.

Borderie, V.M., Baudrimont, M., Lopez, M., et al., 1997. Evaluation of the deswelling period in dextran-containing medium after corneal organ culture. Cornea 16, $215-223$.

Dalimier, E., Salomon, D., 2012. Full-field optical coherence tomography: a new technology for 3D high-resolution skin imaging. Dermatology 224, 84-92. http://dx.doi.org/10.1159/000337423.

Davanger, M., Evensen, A., 1971. Role of the pericorneal papillary structure in renewal of corneal epithelium. Nature 229, 560-561. http://dx.doi.org/10.1038/ $229560 a 0$.

Deng, S., Sejpal, K., Tang, Q., et al., 2012. Characterization of limbal stem cell deficiency by in vivo laser scanning confocal microscopy. Arch. Ophthalmol. 130 (4), 440-445. http://dx.doi.org/10.1001/archophthalmol.2011.378.

Dua, H., Shanmuganathan, V., Powell-Richards, A., et al., 2005. Limbal epithelial crypts: a novel anatomical structure and a putative limbal stem cell niche. Br. J. Ophthalmol. 89, 529-532. http://dx.doi.org/10.1136/bjo.2004.049742.

Dua, H.S., Kulkami, B., Singh, R., 2006. Quest for limbal stem cells. Clin. Exp. Ophthalmol. 34, 1-2. http://dx.doi.org/10.1111/j.1442-9071.2006.01163.x.

Dubois, A., Vabre, L., Boccara, A., et al., 2002. High-resolution full-field optical coherence tomography with a Linnik microscope. Appl. Opt. 41, 805-812. http://dx.doi.org/10.1364/A0.41.000805.

Dziasko, M.A., Armer, H.E., Levis, H.J., et al., 2014. Localisation of epithelial cells capable of Holoclone formation in vitro and direct interaction with stromal cells in the native human limbal crypt. PLoS One 9 (4), e94283. http://dx.doi.org/ 10.1371 /journal.pone.0094283.

Eberwein, P., Reinhard, T., 2014. The role of biomechanics in the limbal stem cell niche: new insights for our understanding of this structure. Stem Cells 20. http://dx.doi.org/10.1002/stem.1886 [Epub ahead of print].

Ghouali, W., Grieve, K., Bellefqih, S., et al., 2014. Full-field optical coherence tomography of human donor and pathological corneas. Cur Eye Res 40 (5), 526-534. http://dx.doi.org/10.3109/02713683.2014.935444.

Ghoubay-Benallaoua, D., Sandali, O., Goldschmidt, P., et al., 2013. Kinetics of expansion of human limbal epithelial progenitors in primary culture of explants with no feeders. PLoS One 8 (12), e81965. http://dx.doi.org/10.1371/ journal.pone.0081965.

Goldberg, M.F., Bron, A.J., 1982. Limbal palisades of Vogt. Trans. Am. Ophthalmol. Soc. $80,155-171$.

Grieve, K., Palazzo, L., Dalimier, E., et al., 2014. A feasibility study of full-field optical coherence tomography for rapid evaluation of EUS-guided microbiopsy specimens. Gastrointest. Endosc. 81 (2), 342-350. http://dx.doi.org/10.1016/ j.gie.2014.06.037.

Grieve, K., Paques, M., Dubois, A., et al., 2004. Ocular tissue imaging using ultrahighresolution, full-field optical coherence tomography. Invest Ophthalmol. Vis. Sci. 45, 4126-4131. http://dx.doi.org/10.1167/iovs.04-0584.

Grieve, K., Dubois, A., Simonutti, M., et al., 2005. In vivo anterior segment imaging in the rat eye with high speed white light full-field optical coherence tomography. Opt. Exp. 13 (16), 6286-6295. http://dx.doi.org/10.1364/OPEX.13.006286.

Haaskjold, E., Olsen, W.M., Bjerknes, R., et al., 1993. Early cell kinetic effects of a single dose of narrow-banded ultraviolet $\mathrm{B}$ irradiation on the rat corneal epithelium. Photochem. Photobiol. 57, 663-666. http://dx.doi.org/10.1111/ j.1751-1097.1993.tb02933.x.

Harms, F., Dalimier, E., Vermeulen, P., et al., January 2012. Multimodal full-field optical coherence tomography on biological tissue: toward all optical digital pathology. In: Proceedings of Multimodal Biomedical Imaging VII, San Francisco, USA, 21-26. http://dx.doi.org/10.1117/12.908459. Proc SPIE 8216.

Huang, D., Swanson, E.A., Lin, C.P., et al., 1991. Optical coherence tomography. Science 254 (5035), 1178-1181. http://dx.doi.org/10.1126/science.1957169.

Lathrop, K., Gupta, D., Kagemann, L., et al., 2012. Optical coherence tomography as a rapid, accurate, noncontact method of visualizing the Palisades of Vogt. IOVS 53 (1), 1381-1387. http://dx.doi.org/10.1167/iovs.11-8524.
Levis, H., Massie, I., Dziasko, M., et al., 2013. Rapid tissue engineering of biomimetic human corneal limbal crypts with 3D niche architecture. Biomaterials 34 8860-8868. http://dx.doi.org/10.1016/j.biomaterials.2013.08.002.

Majo, F., Rochat, A., Nicholas, M., et al., 2008. Oligopotent stem cells are distributed throughout the mammalian ocular surface. Nature 456, 250-254. http:/ dx.doi.org/10.1038/nature07406.

Miri, A., Al-Aqaba, M., Otri, A.M., et al., 2012. In vivo confocal microscopic features of normal limbus. Br. J. Ophthalmol. 96, 530-536. http://dx.doi.org/10.1136 bjophthalmol-2011-300550.

Molvær, R.K., Andreasen, A., Heegaard, S., et al., 2013. Interactive 3D computer model of the human corneolimbal region: crypts, projections and stem cells. Acta Ophthalmol. 91 (5), 457-462. http://dx.doi.org/10.1111/j.1755 3768.2012.02446.x.

Morrison, S.J., Shah, N.M., Anderson, D.J., 1997. Regulatory mechanisms in stem cell biology. Cell 88, 287-298. http://dx.doi.org/10.1016/S0092-8674(00)81867-X.

Nakamura, T., Inatomi, T., Sotozono, C., et al., 2004. Successful primary culture and autologous transplantation of corneal limbal epithelial cells from minimal biopsy for unilateral severe ocular surface disease. Acta Ophthalmol. Scand. 82 468-471. http://dx.doi.org/10.1111/j. 1395-3907.2004.00285.x.

Nakamura, T., Ang, L.P., Rigby, H., et al., 2006. The use of autologous serum in the development of corneal and oral epithelial equivalents in patients with Stevens-Johnson syndrome. Invest. Ophthalmol. Vis. Sci. 47, 909-916. http:// dx.doi.org/10.1167/iovs.05-1188.

Notara, M., Schrader, S., Daniels, J.T., 2011. The porcine limbal epithelial stem cell niche as a new model for the study of transplanted tissue-engineered human limbal epithelial cells. Tissue Eng. Part A 17 (5-6), 741-750.

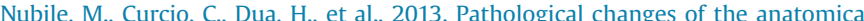
structure and markers of the limbal stem cell niche due to inflammation. Mol. Vis. 19, 516-525.

Patel, D.V., Sherwin, T., McGhee, C.N., 2006. Laser scanning in vivo confocal microscopy of the normal human corneoscleral limbus. Invest. Ophthalmol. Vis. Sci. 47, 2823-2827. http://dx.doi.org/10.1167/iovs.05-1492.

Rama, P., Matuska, S., Paganoni, G., et al., 2010. Limbal stem-cell therapy and longterm corneal regeneration. N. Engl. J. Med. 363 (2), 147-155. http://dx.doi.org/ 10.1056/NEJMoa0905955.

Shanmuganathan, V., Foster, T., Kulkarni, B., et al., 2007. Morphological characteristics of the limbal epithelial crypt. Br. J. Ophthalmol. 91, 514-519. http:/ dx.doi.org/10.1136/bjo.2006.102640.

Shortt, A., Secker, G., Munro, P., et al., 2007. Characterization of the limbal epithelial stem cell niche: novel imaging techniques permit in vivo observation and targeted biopsy of limbal epithelial stem cells. Stem Cells 25, 1402-1409. http:/ dx.doi.org/10.1634/stemcells.2006-0580.

Schlötzer-Schrehardt, U., Kruse, F.E., 2005. Identification and characterization of limbal stem cells. Exp. Eye Res. 81 (3), 247-264. http://dx.doi.org/10.1016/ j.exer.2005.02.016.

Schlötzer-Schrehardt, U., Dietrich, T., Saito, K., et al., 2007. Characterization of extracellular matrix components in the limbal epithelial stem cell compartment. Exp. Eye Res. 85 (6), 845-860. http://dx.doi.org/10.1016/ j.exer.2007.08.020.

Townsend, W.M., 1991. The limbal palisades of Vogt. Trans. Am. Ophthalmol. Soc. 89, 721-756.

Tseng, S.C.G., 1996. Regulation and clinical implications of corneal epithelial stem cells. Mol. Biol. Rep. 23, 47-58.

Watt, F.M., Hogan, B.L., 2000. Out of Eden: stem cells and their niches. Science 287, 1427-1430. http://dx.doi.org/10.1126/science.287.5457.1427.

Yeung, A., Schlötzer-Schrehardt, U., Kulkarni, B., et al., 2008. Limbal epithelial crypt a model for corneal epithelial maintenance and novel limbal regional variations. Arch. Ophthalmol. 126 (5), 665-669. http://dx.doi.org/10.1001/ archopht.126.5.665.

Yokoo, S., Yamagami, S., Usui, T., et al., 2008. Human corneal epithelial equivalents for ocular surface reconstruction in a complete serum-free culture system without unknown factors. Invest. Ophthalmol. Vis. Sci. 49 (6), 2438-2443. http://dx.doi.org/10.1167/iovs.06-1448. 